\title{
Optimal Design of the Reflector Antenna to Improve Performance of C-Band Quad-Pol ScanSAR Systems
}

\author{
Jung-Hwan $\operatorname{Lim}^{1} \cdot$ Jae Wook Lee ${ }^{1, *} \cdot$ Taek-Kyung Lee ${ }^{1} \cdot$ Sang-Burm Ryu ${ }^{2}$. \\ Hyeon-Cheol Lee ${ }^{2} \cdot$ Sang-Gyu Lee ${ }^{2}$
}

\begin{abstract}
The performance of synthetic aperture radar (SAR) antenna determines the quality of images obtained from the spaceborne SAR system, and thus, SAR antenna should be designed to satisfy the target performance of SAR system. The performance indicators of a SAR system, such as ambiguity-to-signal ratio and resolution, also depend on the SAR operation mode. Therefore, it is important to consider the operation mode when designing an SAR antenna. In this paper, we analyzed the SAR antenna's effect on ScanSAR (scanning synthetic aperture radar) which is a representative wide-swath mode and its quadrature-polarimetric (quad-pol) mode. In addition, we confirmed that the optimal reflector antenna for the C-band quad-pol ScanSAR system can be designed by analyzing the relation between the antenna size and the system performance.
\end{abstract}

Key Words: Ambiguity-to-Signal Ratio, Quad-Pol SAR, Reflector Antenna, Resolution, ScanSAR.

\section{INTRODUCTION}

The synthetic aperture radar (SAR) antenna, which affects the performance of the spaceborne SAR system, is an important part of the satellite payload's size and weight. Several factors can affect the performance of the SAR antenna and should be considered when designing it; these factors include the SAR operation mode as well as SAR system performance indicators, such as ambiguity-to-signal ratio (ASR) and resolution. ScanSAR (scanning synthetic aperture radar), which is a representative wide-swath SAR operation mode, can reduce the revisit time of a satellite by observing a wide area. However, the resolution is degraded in proportion to the number of sub-swaths of ScanSAR system [1]. In the case of the quadrature-polarimetric (quad-pol) mode, horizontal/vertical $(\mathrm{H} / \mathrm{V})$ polarization are alternately transmitted and received to obtain various polarization information about the Earth's surface. However, the ambiguity performance of the conventional quad-pol SAR system is worse than single-pol system [2]. For this reason, considering the performance only in single-pol mode when designing the SAR antenna will limit its ability to achieve the target performance in quad-pol mode [3]. Therefore, the effects of SAR operation mode should be considered in the antenna design to achieve better SAR system performance.

In this paper, we analyzed the requirements of the reflector antenna in the spaceborne ScanSAR system's quad-pol application, and we determined the optimal reflector size to satisfy the target performance by analyzing the SAR system performance

Manuscript received July 1, 2019 ; Revised November 7, 2019 ; Accepted December 4, 2019. (ID No. 20190701-052J)

${ }^{1}$ Department of Electronics and Information Engineering, Korea Aerospace University, Goyang, Korea.

${ }^{2}$ Satellite Payload Development Division, Korea Aerospace Research Institute, Daejeon, Korea.

"Corresponding Author: Jae Wook Lee (e-mail: jwlee1@kau.ac.kr)

This is an Open-Access article distributed under the terms of the Creative Commons Attribution Non-Commercial License (http://creativecommons.org/licenses/by-nc/4.0) which permits unrestricted non-commercial use, distribution, and reproduction in any medium, provided the original work is properly cited.

(c) Copyright The Korean Institute of Electromagnetic Engineering and Science. All Rights Reserved. 
according to the antenna size while also considering both the single-pol and quad-pol modes.

\section{DESIGN OF THE REFLECTOR ANTENNA FOR THE}

\section{QUAD-POL SCANSAR}

In general, the ambiguity-to-signal ratio consists of the range ambiguity-to-signal ratio (RASR) and azimuth ambiguity-tosignal ratio (AASR). The resolution is characterized in the terms of the range direction and azimuth direction $[4,5]$. The performance indicators of the SAR system, such as ambiguity ratio and resolution, determine the quality of the images obtained from the SAR and are affected by the antenna radiation patterns. In terms of the ambiguity ratio, using a large antenna is advantageous because the signals received from outside the main beam affect the SAR image as ambiguity signals. In terms of the resolution, illuminating a wide angle using a small anten$\mathrm{na}$ is advantageous because the azimuth resolution is inversely proportional to Doppler processing bandwidth (PB), which is related to the antenna azimuth length.

ScanSAR divides a large swath width into multiple sub-swaths and utilizes multi-beam for each sub-swath. Using a reflector antenna with feed arrays enables beam-forming simply by turning the feed antennas on and off. ScanSAR can avoid blind ranges determined by pulse repetition frequency (PRF) and transmitted pulse width and can improve noise performance. However, as the dwell time of each sub-width decreases, the azimuth resolution is degraded in proportion to the number of sub-swaths [1]. Therefore, increasing the antenna width in the elevation direction reduces the beamwidth of the antenna patterns formed by each feed, which discontinuously worsens the resolution because of the increase of the number of the subswaths and feeds for maintaining the total swath width. To avoid changing the focal length of the reflector antenna, the space between feeds, which is related to the beam deviation factor [6], is reduced. Therefore, the size of the feed antenna should be considered in determining the reflector width, even though the resolution constraint does not limit the antenna design.

The quad-pol SAR mode obtains various polarization signals, such as $\mathrm{HH}, \mathrm{HV}, \mathrm{VH}$, and VV. However, the ambiguity performance is degraded by the combination of the polarization signals, especially in the RASR performance for $\mathrm{HV}$ and $\mathrm{VH}$ [2]. The quad-pol mode can use only a half swath width compared to the single-pol mode because of the ambiguity performance degradation, especially in the high incidence angle region. For this reason, the quad-pol mode has a smaller number of sub-swaths, and it has the advantage of better resolution in ScanSAR mode.

Because of the different characteristics of the two SAR op-

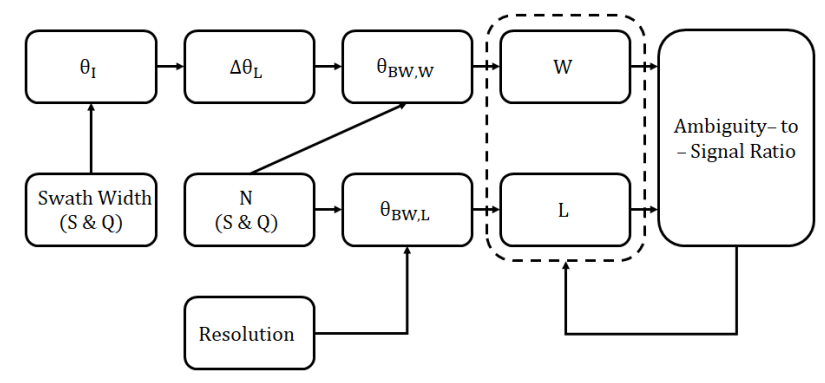

Fig. 1. The relation between the system parameters of ScanSAR. $\mathrm{S}=$ single-pol, $\mathrm{Q}=$ quad-pol.

eration modes (single-pol and quad-pol), the optimal antenna for each mode is different. However, the two cases cannot be considered independently because the same antenna must be used. This means that using a larger antenna improves the ambiguity performance of the quad-pol mode, but its effect on the resolution of single-pol mode must be considered.

Fig. 1 shows the relation between the system parameters of ScanSAR, which affect the antenna design, such as the swath width, the number of sub-widths, $N$, the range of the look angle, $\Delta \theta_{L}$, the antenna elevation/azimuth size, $W / L$, and the antenna beamwidth, $\theta_{B W, W} / \theta_{B W, L}$.

Based on the relation shown in Fig. 1, the reflector size was selected by analyzing the ambiguity performance of different antenna widths and lengths.

\section{PERFoRmance ANALYSIS RESUlts of the}

\section{QUAD-POL SCANSAR SYSTEM}

In this paper, we assumed the C-band ScanSAR system used an offset reflector antenna at a satellite altitude of $505 \mathrm{~km}$ [4]. The performance of the quad-pol ScanSAR system was analyzed according to the size of the reflector antenna by applying the antenna patterns obtained by FEKO simulation tool. ScanSAR has a swath width of $150 \mathrm{~km}$ in single-pol mode, and the $\mathrm{PRF}$ and $\mathrm{PB}$ were determined considering the SAR system performance [3].

We verified the performance analysis procedure of the quadpol SAR system and obtained results similar to those of previous work [3]. The analysis results of the ambiguity performance presented in this chapter were obtained by this procedure.

As an example of performance analysis, the analysis results of the ambiguity performance of a $4 \mathrm{~m}(\mathrm{~L}) \times 1.35 \mathrm{~m}(\mathrm{~W})$ reflector antenna are shown in Fig. 2. In this case, the single-pol mode had a better ambiguity performance than the quad-pol mode at the range of incidence angle with which we are concerned. Although quad-pol mode had the less PB because of the azimuth ambiguity performance, the two modes had a similar resolution because quad-pol mode had a less swath width under the criterion of $-20 \mathrm{~dB}$ of the ambiguity ratio. 


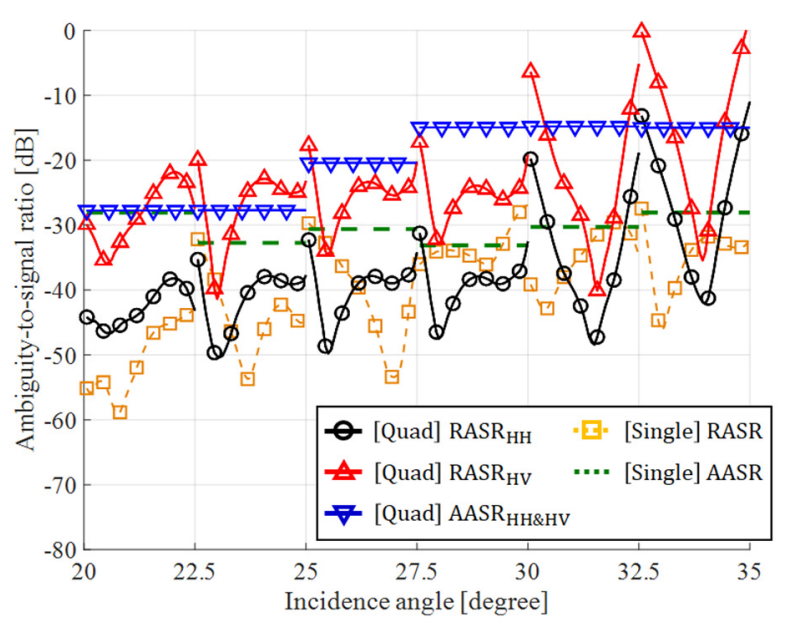

Fig. 2. The ambiguity performances of the ScanSAR system.

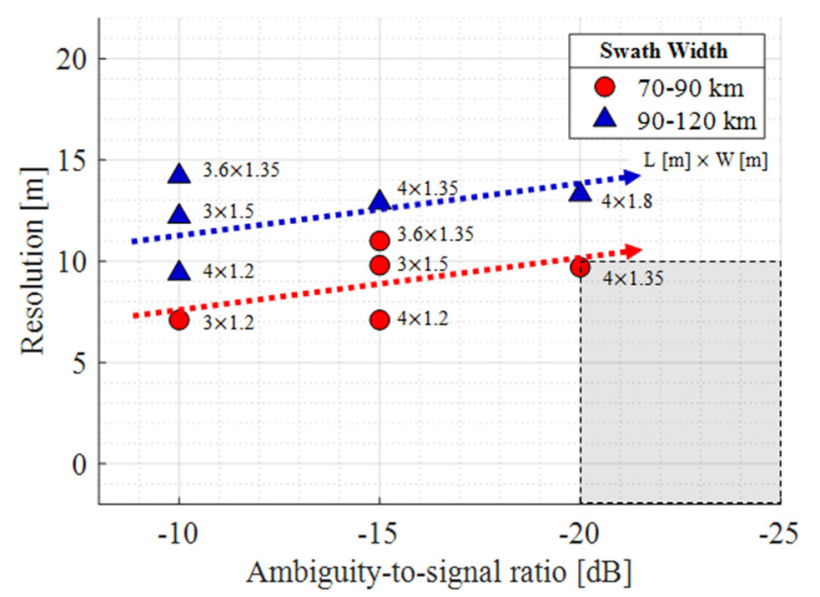

Fig. 3. Relation between the ambiguity-to-signal ratio and the resolution, according to the reflector antenna size, in the Cband quad-pol ScanSAR system.

Fig. 3 shows the performance comparison result with various antenna sizes. As shown in Fig. 3, we can confirm the trade-off relation among the antenna size, ambiguity ratio, resolution, and swath width as mentioned in Section $\Pi$. In addition, if the target performances are RASR/AASR under $-20 \mathrm{~dB}$ and resolution under $10 \mathrm{~m}$, a reflector size of $4 \mathrm{~m} \times 1.35 \mathrm{~m}$ is the antenna optimized to satisfy the requirement of the ScanSAR system.

\section{CONCLUSION}

In this paper, the reflector antenna that is optimal for both the single-pol and quad-pol modes of C-band ScanSAR system was designed by analyzing the relation between antenna size and SAR system performance. Based on the simulation results, we conjectured that the range of performance, which the ScanSAR system can obtain under a SAR operation scenario, can be estimated based on the trend of the ambiguity ratio and the resolution produced by variations in the antenna size.

This work was performed for the Development of Radar Payload Technologies for Compact Satellite in Korea Aerospace Research Institute, funded by the Ministry of Science and ICT.

\section{REFERENCES}

[1] D. D'Aria, F. D. Zan, D. Giudici, A. M. Guarnieri, and F. Rocca, "Burst-mode SARs for wide-swath surveys," Canadian Journal of Remote Sensing, vol. 33, no. 1, pp. 27-38, 2007.

[2] M. Villano, G. Krieger, and A. Moreira, "New insights into ambiguities in quad-pol SAR," IEEE Transactions on Geoscience and Remote Sensing, vol. 55, no. 6, pp. 3287-3308, 2017.

[3] J. H. Lim, S. S. Yoon, J. W. Lee, T. K. Lee, S. B. Ryu, H. C. Lee, and S. G. Lee, "Performance analysis of quad-pol SAR system for wide-swath operation mode," The Journal of Korean Institute of Electromagnetic Engineering and Science, vol. 30, no. 2, pp. 141-151, 2019.

[4] S. S. Yoon, J. W. Lee, T. K. Lee, S. B. Ryu, H. C. Lee, E. S. Kang, and S. G. Lee, "System performance analysis for multi-band sweepSAR operating mode," The Journal of Korean Institute of Electromagnetic Engineering and Science, vol. 28, no. 3, pp. 186-194, 2017.

[5] I. G. Cumming, and F. H. Wong, "Synthetic aperture concepts," in Digital Processing of Synthetic Aperture Radar Data: Algorithms and Implementation. Boston, MA: Artech House, 2005, pp. 113-168.

[6] W. L. Stutzman, and G. A. Thiele, Antenna Theory and Design, 3rd ed. New York, NY: John Wiley \& Sons, 2013, pp. 407-410. 